\title{
Reconstruction Method of Electrical Capacitance Tomography Based on Wavelet Fusion
}

\author{
Zhou Lei ${ }^{1,2, *}$, Yan Jiangbao ${ }^{2,3}$, Zhu Feng $^{1}$, Tan Xiangyu ${ }^{2}$,and Zhang Lifeng ${ }^{1}$ \\ ${ }^{1}$ Department of Automation, North China Electric Power University,071003,China \\ ${ }^{2}$ Yunnan Electric Power Research Institute,Kunming,650217, China \\ ${ }^{3}$ College of Information Engineering and Automation, Kunming University of Science and Technology, Kunming, 650500, China
}

\begin{abstract}
The accuracy of reconstructed images of Electrical Capacitance Tomography (ECT) is a bottleneck concerning the successful application of ECT. An image data fusion algorithm based on wavelet transform was proposed in this paper to improve the accuracy of reconstructed images. First, reconstructed images were obtained using conjugate gradient least square algorithm and Landweber iterative algorithm, respectively. Secondly, reconstructed images were decomposed by wavelet. After that, the approximate component was processed according to the weighted average fusion rule. The detail component was processed according to the maximum fusion rule of absolute value. Finally, the new reconstructed images were obtained by wavelet reconstruction. Simulation and static experimental results showed that the reconstructed images with higher accuracy can be obtained after fusion and the artefacts were decreased obviously.
\end{abstract}

\section{Introduction}

Electrical capacitance tomography (ECT) is a kind of process tomography technique that was developed during the late 1980s. The ECT technique has been investigated extensively during the past decades as a visualization technique for measurement and imaging of two-phase flows in real time in industrial process monitoring. ECT is considered to be one of the most attractive process imaging technology with wide developing prospect due to its advantages of noninvasion, high-speed, safety and low cost $[1,2]$.

The task of image reconstruction for ECT is to determine the permittivity distribution and hence material distribution over the cross-section using the capacitance measurements which were obtained by data acquisition system (DAS) from different viewing angle via array electrodes which are mounted circumferentially around an industrial flow pipe or receptacle and relies on changes in capacitance between electrodes owing to the change in permittivity of flow components.

ECT image reconstruction is a typical ill-posed problem and its solution is unstable. Image algorithms of ECT can be divided into non-iterative and iterative algorithms [3]. Non-iterative algorithms are commonly fast in speed. The most commonly used non-iterative algorithms are linear back projection (LBP) and Tikhonov regularization algorithms. On the other hand, the most commonly used iterative algorithms contain Landweber iterative algorithm and conjugate gradient least square (CGLS) iterative algorithm. Non-iterative algorithm is simple and fast, but the algorithm is poor in image quality and is generally used for qualitative analysis.

Landweber iterative algorithm is similar to the steepest gradient method, and its convergence is poor for the complicated flow patterns, which limits its application [4]. CGLS algorithm is ideal for simple flow patterns and complex flow patterns [5]. In order to further improve the accuracy of the reconstructed images, an image fusion method based on wavelet decomposition was presented. First, reconstructed images were obtained by Landweber iterative algorithm and CGLS algorithm, respectively. Secondly, wavelet transforms were carried out on these reconstructed images and the corresponding image fusion method was applied on it. Finally, images were obtained using wavelet reconstruction. Simulation and static experiments were carried out and the results showed that the quality of reconstructed images was improved.

\section{Principle of ECT}

\subsection{ECT System}

The diagram of a16-electrode ECT system is shown in Fig. 1. Capacitances are measured between different electrode pairs and the measurements obtained are used to reconstruct a cross sectional distribution of flow components.

\footnotetext{
*Corresponding author: Zhou Lei: zhouleiect@163.com
} 


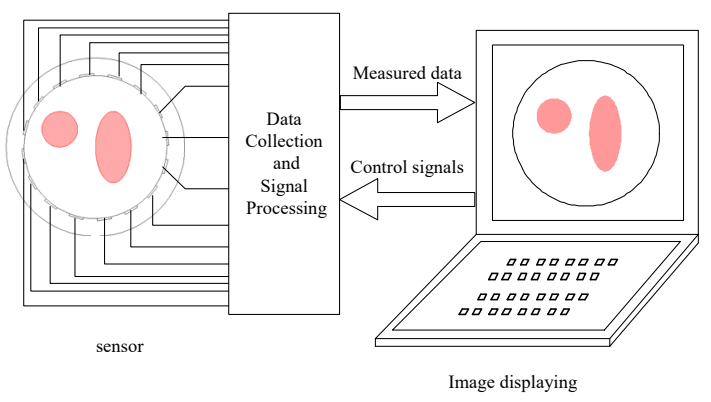

Fig. 1. Structure of 16-electrode ECT system

For a 16-electrode system, the number of independent capacitance measurements is 120. ECT is composed of forward problem and inverse problem. The forward problem is to determine capacitance measurements given the permittivity distribution and the boundary condition. The inverse problem is also called image reconstruction.

\subsection{Image Reconstruction Algorithm of ECT}

ECT image reconstruction algorithms are based on the linear model of the mapping of permittivity distribution to capacitance values. After being discrete, linearized and normalized, the model is shown in (1):

$$
\mathbf{C}=\mathbf{S G}
$$

Where $\mathbf{C}$ is the normalized capacitance vector, $\mathbf{S}$ is the sensitivity matrix of normalized capacitance with respect to normalized permittivity and $\mathbf{G}$ is the normalized permittivity vector.

In this paper, Landweber iterative algorithm and CGLS algorithm are selected to obtain the initial reconstructed images.

\subsection{Landweber Iterative Algorithm}

The steepest descent method selects the negative gradient direction as the iterative direction, and the iterative formula of the Landweber iterative algorithm is shown in (2):

$$
\left\{\begin{array}{l}
\mathbf{G}_{k+1}=\mathbf{G}_{k}+\alpha \mathbf{S}^{T}\left(\mathbf{C}-\mathbf{S G}_{k}\right) \\
\mathbf{G}_{0}=\mathbf{S}^{T} \mathbf{C}
\end{array}\right.
$$

Where $\alpha$ is the step length and $\mathbf{G}_{k}$ is the normalized image grey in the kth iteration. $\mathbf{G}_{0}$ is the initial value of the image grey.

\subsection{CGLS Algorithm}

Conjugate Gradient Least Squares (CGLS) is developed by Conjugate Gradient (CG) algorithm. It is an excellent iterative algorithm for solving large dimensionality illposed systems and the system matrix can be asymmetric and singular. The basic idea of the CGLS iterative regularization algorithm is to apply the conjugate gradient method to solving the problem of the minimum (3):

$$
\min _{\mathbf{G}_{m}}\left\|\mathbf{C}-\mathbf{S G}_{m}\right\|_{2}
$$

It is required for the $\mathrm{CG}$ iterative algorithm that the sensitivity matrix must be symmetric positive definite. We multiply the two sides of (3) to obtain the normal equation:

$$
\mathbf{S}^{T} \mathbf{S G}=\mathbf{S}^{T} \mathbf{C}
$$

And then, the sensitivity matrix $\mathbf{A}=\mathbf{S}^{T} \mathbf{S}$ is symmetric positive definite. Conjugate gradient method is used for CGLS algorithm to solve the asymmetric problem and hence the normal equation can be obtained. The CGLS algorithm is carried out according to the following steps. $m=1$

Initialization: $\mathbf{G}_{0}=0, r_{0}=\mathbf{C}-\mathbf{S G}_{0}, d_{0}=\mathbf{S}^{T} r_{0}$,

$$
\begin{aligned}
& \alpha_{m}=\frac{\left\|\mathbf{S}^{T} r_{m-1}\right\|_{2}^{2}}{\left\|\mathbf{S} d_{m-1}\right\|_{2}^{2}} \\
& \text { step 1: } \mathbf{G}_{m}=\mathbf{G}_{m-1}+\alpha_{m} d_{m-1} \\
& \text { step 2: } r_{m}=r_{m-1}-\alpha_{m} \mathbf{S} d_{m-1} \\
& \text { step 3: } \\
& \text { step 4: } \quad \beta_{m}=\frac{\left\|\mathbf{S}^{T} r_{m}\right\|_{2}^{2}}{\left\|\mathbf{S} r_{m-1}\right\|_{2}^{2}} \\
& \text { step 5: } d_{m}=\mathbf{S}^{T} r_{m}+\beta_{m} d_{m-1} \\
& m=m+1
\end{aligned}
$$

Where $m$ is the iteration step, $\alpha_{m}$ is the iteration step size, ${ }^{r}$ is the residual vector, $\beta_{m}$ is the conjugate coefficient and $d_{m}$ is the iteration search direction, respectively.

It can be seen from the above algorithm steps that the CGLS iteration process involves only the product of the vector and the sensitivity matrix or the transposed matrix, and the iteration step $m$ is used as the regularization parameter. As the CGLS algorithm has a semiconvergence, the inappropriate iterative step size will cause "under estimate" or "over estimate" results.

\section{Image Reconstruction Method for ECT Based on Wavelet Fusion}

The reconstructed images have artefacts or noise, and the spatial resolution is not high. In order to further improve the image quality, the reconstructed image is processed by wavelet fusion. Wavelet analysis is a tool for signal multiresolution analysis, which has good properties in both time and frequency domain.

\subsection{Wavelet Transform for the Reconstructed Images}


To fulfill the wavelet fusion for the reconstructed images which were used as the source images, wavelet decomposition for the source images was carried out. After that, high and low frequency coefficients of each source image can be obtained. And then, image fusion based on the regional features of the decomposed wavelet coefficient matrix was adopted. Finally, the new reconstructed images are obtained by inverse wavelet transform. After wavelet transform, the total data of the image will not increase. The wavelet transform is nonredundant and wavelet transform is directional, which makes it widely used in the field of image fusion [6].

Assuming the function $f(t) \in L^{2}(R)$, the wavelet transform is defined as (11):

$$
\begin{aligned}
W_{f}(a, b) & =\int_{-\infty}^{+\infty} f(t) \phi_{a, b}(t) d t \\
& =\int_{-\infty}^{+\infty} f(t) \frac{1}{\sqrt{a}} \phi\left(\frac{t-b}{a}\right) d t
\end{aligned}
$$

Where $\phi_{a, b}(t)$ is the basis function, $a$ is the scale parameter $(a>0)$ and $b$ is the translation parameter, respectively.

In the image processing, it is necessary to discretize the continuous wavelet, which is also known as discrete wavelet transform (DWT). In general, the discrete formula of the scale parameter $a$ and the translation parameter $b$ in the continuous wavelet transform are taken as $a=a_{0}^{m}$ and $b=n a_{0}^{m} b_{0} \quad\left(m \in Z, a_{0}>1\right)$. And then, the corresponding discrete wavelet function $\phi_{m, n}(t)$ can be written as (12):

$$
\begin{aligned}
\phi_{m, n}(t) & =\frac{1}{\sqrt{a_{0}^{m}}} \phi\left(\frac{t-n b_{0} a_{0}^{m}}{a_{0}^{m}}\right) \\
& =a_{0}^{-m / 2} \phi\left(a_{0}^{m} t-n b_{0}\right)
\end{aligned}
$$

The corresponding discrete wavelet transform coefficients can be defined by (13):

$$
\begin{aligned}
C_{m, n} & =\int_{-\infty}^{+\infty} f(t) \phi_{m, n}^{*}(t) d t \\
& =<f, \phi_{m, n}>
\end{aligned}
$$

Reconstruction formula of wavelet transform is (14):

$$
f(t)=\alpha \sum_{-\infty}^{+\infty} \sum_{-\infty}^{+\infty} C_{m, n} \phi_{m, n}(t)
$$

Where $\alpha$ is a signal-independent constant.

\subsection{Image Fusion Rules}

Three are three levels in image fusion: pixel level, feature level and decision level. In this paper, pixel level image fusion method was used to improve the accuracy of the reconstructed images [7]. The process of this image fusion method is as follows:

step 1: source images are obtained using Landweber iterative algorithm and CGLS algorithm, respectively. step 2: filtering processing: a mean filter is used to the image pixels.

step 3: The wavelet decomposition is carried out by choosing the appropriate wavelet basis function and decomposition level.

step 4: The fusion process of the approximate component and the detail component is made beads on the corresponding fusion rule, respectively.

step 5: Wavelet reconstruction based on the wavelet coefficients after fusion is completed and the final reconstructed images can be obtained.

Fig. 2 shows the structural diagram of the image fusion method based on wavelet transform.



Fig. 2. Image Fusion Process Based on Wavelet Transform

In this paper, the fusion rules and fusion algorithms used are as follows:

Rule 1: The approximation component of the fusion image is processed by the improved logic filter algorithm fusion rule, as shown in (15):

$$
\mathbf{X}_{\mathbf{M}}(x, y)=\theta \mathbf{M}_{1}(x, y)+(1-\theta) \mathbf{M}_{2}(x, y)
$$

Where $\theta$ is the weighting coefficient and let $\theta=0.5$. $\mathbf{M}_{1}(x, y)$ and $\mathbf{M}_{2}(x, y)$ is the approximate component of the wavelet decomposition of the reconstructed images obtained by Landweber iterative and CGLS algorithm, respectively. The approximate component contains the main information of the image. The average characteristics of the source images can be reflected by this part, which reflects the overall outline of the image. The low frequency part is fused by the weighted averaging operator.

Rule 2: The detail component of the fusion image is processed by the fusion rule with the largest absolute value of the coefficient, as shown in (16):

$$
\mathbf{Y}_{\mathbf{N}}(x, y)=\max \left\{\left|\mathbf{N}_{1}(x, y)\right|,\left|\mathbf{N}_{2}(x, y)\right|\right\}
$$

Where $\mathbf{N}_{1}(x, y)$ and $\mathbf{N}_{2}(x, y)$ is the detail component of the wavelet decomposition of the reconstructed images obtained by Landweber iterative and CGLS algorithm, respectively. The details of source images such as edges and regional boundary features are mainly reflected by the detail component part. And hence the dealing for the high frequency part is the key of the whole fusion process. The fusion rule based on the largest absolute value of the coefficient is adopted for the detail component part. 


\section{Experimental Results and Analysis}

\subsection{Simulation Experiment}

In the simulation experiment, oil-gas two-phase flow was selected as the research object and 4 typical flow phantoms were studied. The relative permittivity of the oil and gas is set to 3 and 1, respectively. The COMSOL finite element software was used to obtain the simulated capacitance measurement data and the ECT image reconstruction was completed by Matlab software. The imaging area is divided into 812 pixels.

In simulation process, the Landweber algorithm and the CGLS algorithm are used as the image reconstruction algorithm to obtain the initial reconstructed image, and then the wavelet transform theory is used as the image fusion algorithm to further improve the image precision. Among them, the Landweber algorithm and the CGLS algorithm take 100 and 25 iterations respectively. In this process, the "bior3.7" function is chosen as the base function of the wavelet transform, and the decomposition level is 3. The simulation results are shown in Fig3.



Fig. 3. Image reconstruction results

The image quality is calculated for quantitative evaluation, and the relative error and correlation coefficient are calculated. The definition is shown in Ref. [3].

The quantitative index $\mathrm{RE}$ and $\mathrm{CC}$ of the reconstructed images of the reconstructed images in Fig. 3 are calculated, which can be seen in Table 1 and Table 2 , respectively.

Table 1. Image relative error

\begin{tabular}{|c|c|c|c|}
\hline Flow Phantom & Landweber & CGLS & Fusion image \\
\hline$a$ & 0.7931 & 0.5454 & 1.5543 \\
\hline$b$ & 0.7532 & 0.7106 & 1.1342 \\
\hline$c$ & 0.5821 & 0.6130 & 1.3169 \\
\hline$d$ & 0.4288 & 0.5341 & 0.9540 \\
\hline
\end{tabular}

Table 2. Correlation coefficients

\begin{tabular}{|c|c|c|c|}
\hline Flow Phantom & Landweber & CGLS & Fusion image \\
\hline$a$ & 0.5957 & 0.8749 & 0.3130 \\
\hline$b$ & 0.7488 & 0.7666 & 0.4345 \\
\hline$c$ & 0.9277 & 0.7156 & 0.2963 \\
\hline$d$ & 0.8817 & 0.7657 & 0.4234 \\
\hline
\end{tabular}

It can be seen from Fig. 3 and Tables I and II that the quality of reconstructed images after wavelet fusion is improved for the selected 4 flow phantoms. As to phantom $b$, the two objects can be separated clearly after image fusion and have better shape fidelity, while there are artefacts in the reconstructed images using Landweber iterative or CGLS algorithm. For phantom c, the reconstructed image was greatly improved with fewer artefacts and better fidelity. Due to the low sensitivity on the center of the pipe, the reconstructed images of phantoms a and $\mathrm{d}$ after wavelet fusion is slightly improved. But it still can be seen that for the complicated distribution phantom d, objects also can be separated more clearly and better shape fidelity can be obtained compared with that of Landweber iterative or CGLS algorithm.

\subsection{Static Experiment}

Static experiment was carried out using the digital ECT system developed by Tianjin University [8], which can be seen in Fig. 4. The ECT system contains 24 channels, which was designed for two plane of 12-electrode sensor. It also can be used according to custom-defined scheme. In our static experiment, we use 16 channels for the 16electrode sensor.

In the static two-phase flow experiment, a 16electrode sensor pipe with the inner diameter of $125 \mathrm{~mm}$ was used. In low pattern I, the pipe is divided into two parts with a plastic plate, half of which is filled with plastic particles. As to flow pattern II, there are two hollow plastic rods with the diameter of $15 \mathrm{~mm}$. The image area is also divided into 812 pixels.

The static experimental sensor and the results were showed in Fig. 5.

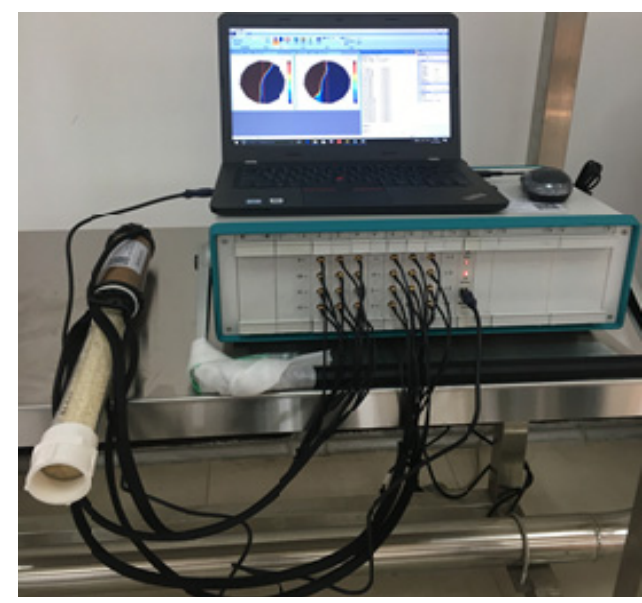

Fig. 4. Digital ECT system 


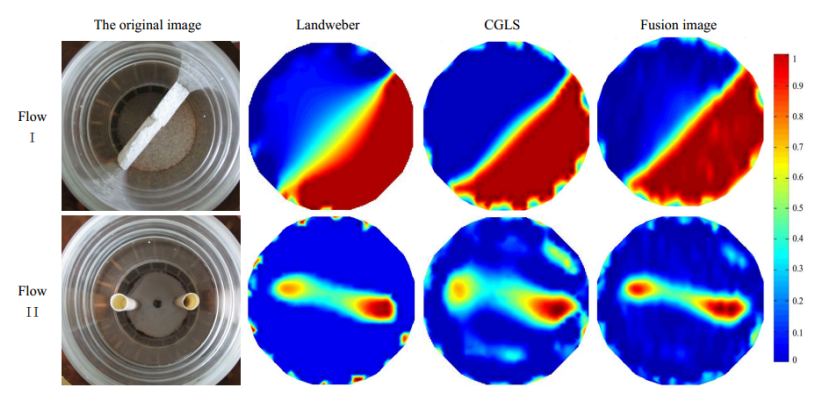

Fig.5. Experimental imaging results

For the flow pattern I, the separation of gas and plastic particles in the reconstructed image after image fusion is much clearer and more accurate than that of Landweber iterative or CGLS algorithm. As to flow pattern II, the two plastic tubes can be clearly distinguished and the image artefacts can be reduced obviously, which fully demonstrates the effectiveness of the fusion method based on wavelet transform.

\section{Conclusions}

In this paper, an ECT image fusion method based on wavelet transform is proposed. Landweber iterative and the CGLS algorithms were used to obtain the source images, which were used for image fusion using wavelet transform. Simulation and static experiments were carried out. Experimental results showed that the accuracy of reconstructed image is improved and the higher edge fidelity of the object can be obtained, which provides a new method for the study of ECT image reconstruction.

\section{Acknowledgment}

The author would like to thank Dr. Lifeng Zhang for his helpful suggestions on the use of ECT DAS during the static experiments.

\section{References}

1. H. S. Tapp and R. A. Williams, "Chemical engineering applications of electrical process tomography,” Chem. Eng. J., vol. 77, pp. 119-125, 2000 .

2. I. Ismail, J. C. Gamio, S. F. A. Bukhari and W. Q. Yang, "Tomography for multi-phase flow measurement in the oil industry," Flow Meas. Instrum., vol. 16, pp. 145-155, 2005.

3. W. Q. Yang and L. H. Peng, "Image reconstruction algorithms for electrical capacitance tomography," Meas. Sci. \& Technol., vol. 14, pp. R1-R13, 2003.

4. W. Q. Yang, D. M. Spink, T. A. York and H. McCann, "An image-reconstruction iteration method for electrical-capacitance tomography," Meas. Sci. \& Technol., vol. 10, pp. 1065-1069, 1999.

5. H. X. Wang, X. M. Zhu and L. F. Zhang, "Conjugate gradient algorithm for electrical capacitance tomography," Journal of Tianjin University, vol. 38, pp. 1-4.

6. P. J. Zheng and J. W. Huang, "Discrete Wavelet Transform and Data Expansion Reduction in Homomorphic Encrypted Domain," IEEE Trans. Image Process., vol. 22, pp. 2455-2468, 2013.

7. P. F. Chai, X. Q. Luo and Z. C. Zhang, "Image Fusion Using Quaternion Wavelet Transform and Multiple Features," IEEE Access, vol. 5, pp. 67246734, 2017.

8. H. X. Wang and L. F. Zhang, "Identification of twophase flow regimes based on support vector machine and electrical capacitance tomography," Meas. Sci. \& Technol., vol. 20, pp. 114007(8 pp), 2009. 